\title{
ICA-SVM Combination Algorithm for Identification of Motor Imagery Potentials
}

\author{
Dong Ming, Changcheng Sun, Longlong Cheng*, \\ Yanru Bai, Xiuyun Liu, Xingwei An, Hongzhi Qi, \\ Baikun Wan \\ Department of Biomedical Engineering \\ Tianjin University \\ Tianjin, China \\ *: cenlon@tju.edu.cn
}

\begin{abstract}
Mental tasks such as motor imagery in synchronization with a cue which result event related desynchronization (ERD) and event related synchronization (ERS) are usually studied in brain-computer interface (BCI) system. In this paper we analyze and classify the ERD/ERS response evoked by the motor imagery of left hand, right hand, foot and tongue. The signals were spatially filtered by Independent Component Analysis (ICA) before calculating the power spectral density (PSD) for related electrodes, and then the Support Vector Machine (SVM) was adopted to recognise the different imagery pattern according to ERD/ERS feature for the signals. The results showed that the combination of ICA-based signal extraction algorithm and SVM-based classification method was an effective tool for the identification of motor imagery potentials, with the highest accuracy rate of $91.4 \%$ and $77.6 \%$ for the lowest.
\end{abstract}

Keywords-Brain-Computer Interface (BCI); Event-Related Desynchronization/Synchronous (ERD/ERS); Support Vector Machine (SVM); Power Spectral Density (PSD); Independent Component Analysis (ICA); ERD/ERS coefficient

\section{INTRODUCTION}

An EEG-based BCI provides a new communication channel between the human brain and a computer. Patients who suffer from severe motor impairments may use such a BCI system as an alternative form of communication by mental activity [1-4]. A good classifier should be designed to achieve a satisfactory communication by mental activity [5].

A BCI with satisfactory classification accuracy depends not only on the good performance of the classifier but also on the choice of proper parameters characterizing EEG signal features [6]. Thus the studies on event-related EEG are meaningful for choosing EEG features and classifying the certain mental tasks. For the ERD/ERS responses, there have been a lot of discussions on the imagination of left and right hand movements with many reliable results for its characteristic frequency band and the corresponding cortical activity in the region of cerebral cortex [7-9]. But the research on the foot and tongue ERD/ERS signals is still limited, their characteristic frequency bands and the corresponding brain regions are still unconvincing. The ERD/ERS derived from motor imagery of

\author{
Yong $\mathrm{Hu}$, KDK Luk \\ Department of Orthopaedics and Traumatology \\ University of Hong Kong \\ Hong Kong, China
}

left hand, right hand, foot and tongue were analyzed in this work using an effective feature extraction method of ICA after PSD, and then SVM was employed for the classification to identify the different motor imagery patterns

\section{ICA-SVM COMBINATION ALGORITHM}

\section{A. Independent Component Analysis (ICA)}

ICA is a blind source separation technique where multichannel signals are assumed to be a linear mixture of independent signal sources. It is a statistical and computational technique for revealing hidden factors that underlie sets of random variables, measurements, or signals [10]. The EEG data, collected with international 10-20 system which is based on the relationship between the location of an electrode and the underlying area of cerebral cortex, was presumably mixed by some underlying components of brain activity. ICA reveals interesting information for brain activity by giving access to its independent components [11]. Assuming that the sources are $\mathbf{s}(\mathbf{t})=\left[\mathrm{s}_{1}(\mathrm{t}), \mathrm{s}_{2}(\mathrm{t}), \ldots, \mathrm{s}_{\mathrm{n}}(\mathrm{t})\right]^{\mathrm{T}}$ and observations are $\mathbf{x}(\mathbf{t})=$ $\left[\mathrm{x}_{1}(\mathrm{t}), \mathrm{x}_{2}(\mathrm{t}), \ldots, \mathrm{x}_{\mathrm{n}}(\mathrm{t})\right]^{\mathrm{T}}$, then the linear mixture can be represented by:

$$
\mathbf{x}(\mathbf{t})=\operatorname{As}(\mathbf{t})
$$

where $\mathbf{A}$ is the mixing matrix. The purpose of ICA is finding a de-mixing matrix such that:

$$
\mathbf{s}(\mathbf{t})=\mathbf{W x}(\mathbf{t})
$$

where $\mathbf{W}=\left[w_{1}, w_{2}, w_{3}, \ldots, w_{\mathrm{n}}\right]$ is the de-mixing matrix.

According to different imaginary movement to decompose the single-stimulus data, independent component $u_{i}$ and demixing matrix $\mathbf{W}$ can be calculated [12]. Then the data after filtering can be represented by:

$$
\left[\begin{array}{l}
x_{1}(t) \\
\vdots \\
x_{i}(t) \\
\vdots \\
x_{N}(t)
\end{array}\right]=W^{-1}\left[\begin{array}{l}
u_{1}(t) \\
\vdots \\
u_{i}(t) \\
\vdots \\
u_{N}(t)
\end{array}\right]=\left(\begin{array}{ccc}
w_{11}^{\prime} & \cdots & w_{1 N}^{\prime} \\
\vdots & \ddots & \vdots \\
w_{N 1}^{\prime} & \cdots & w^{\prime}{ }_{N N}
\end{array}\right]\left[\begin{array}{l}
u_{1}(t) \\
\vdots \\
u_{i}(t) \\
\vdots \\
u_{N}(t)
\end{array}\right]
$$


where, $x_{i}$ is the potential which $u_{i}$ projected onto the scalp electrodes, and $w_{i j}^{\prime}$ are weight coefficient of each projection. Reserve the excellent spatial distribution pattern based on the performance of corresponding components for the four motor imagery patterns, while empty others.

\section{B. Power Spectral Density (PSD)}

The Power Spectral Density (PSD) is defined basing on Short-time Fourier transform (STFT).:

$$
S T F T_{x}(\omega, t)=\int_{-\infty}^{\infty} x\left(t^{\prime}\right) w^{*}\left(t^{\prime}-t\right) e^{-j \omega t^{\prime}} d t^{\prime}
$$

where, "*"is the conjugate of complex, $w(t)$ is the window function, $x(t)$ is the signal to be transformed. For a certain fixed time $t$, the frequency information in $\left[t-D_{t} / 2, t+D_{t} / 2\right]\left(D_{t}\right.$ is the effective time width of $w(t))$ of the signal is given by STFT.

The PSD of the signal is given by:

$$
S_{x}(\omega, t)=\left|S T F T_{x}(\omega, t)\right|^{2}
$$

The ERD/ERS result from different imaginary movements could be expressed effectively through locating the temporal and frequency characters by PSD that based on Short-time Fourier transform.

\section{Support Vector Machines (SVM)}

The Support Vector Machines (SVM), proposed by Vapnik, is a classifier that performs classification by constructing an Ndimensional hyperplane that optimally separates the data into two categories. The goals of SVM are separating the data with hyper plane and extend this to non-linear boundaries using kernel trick In particular, SVMs, using the idea of kernel substitution, have presented great ability as a classification tools and become increasingly popular recognition method for analyzing EEG signals [13].

The construction of a hyperplane $\mathbf{w}^{T} \mathbf{x}+b=0$ ( $\mathbf{w}$ is the weight vector, $b$ is an offset parameter) was achieved so that the margin between the hyperplane and the nearest point was maximized (see Fig. 1). The only free parameter, $C$, in SVMs controlled the trade-off between the maximization of margin and the amount of misclassifications [14].

Such a SVM enabled classification using linear decision boundary and is known as a "linear SVM". A nonlinear decision boundary can be created with only a low increase of the classifier' s complexity by using the "kernel trick". The nonlinear decision function was given by:

$$
\begin{aligned}
f(x) & =\operatorname{sign}[D(x)] \\
\mathrm{D}(x) & =\sum_{i=1}^{\# S V} \alpha_{i} y_{i}\left(x, x_{i}^{S V}\right)+b
\end{aligned}
$$

where $\mathrm{D}(\mathrm{x})$ was the signed distance function for the evaluation of classification confidence, $\alpha_{i}$ was the nonnegative Lagrange

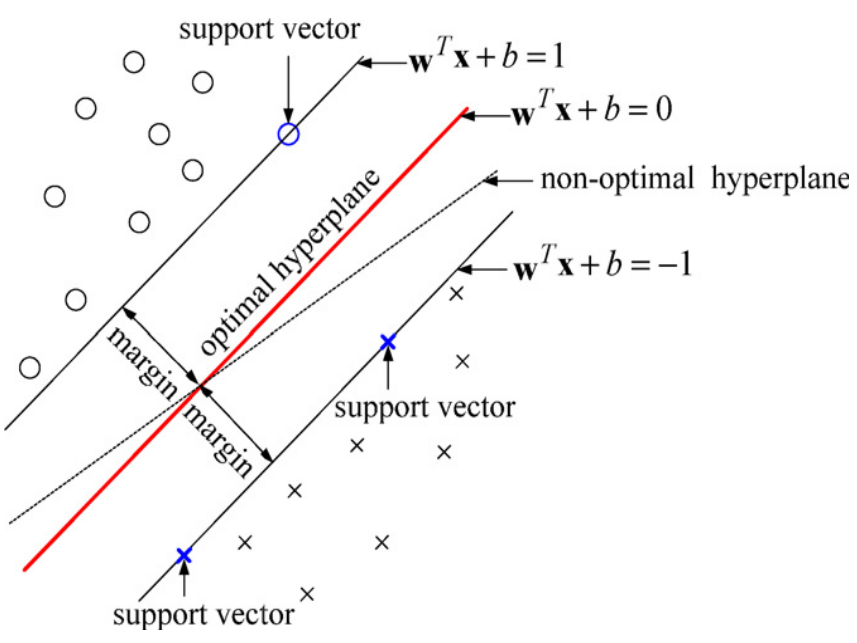

Figure 2. SVM found the optimal hyperplane for generalization. The hyperplane $\mathbf{w}^{\mathrm{T}} \mathbf{x}+\mathrm{b}=0$ separated two classes: the "circles" and the "crosses"

multipliers, \#SV was the number of support vectors, and b was an offset parameter. The data points $x_{i}^{S V}$ corresponding with $\alpha_{i}>0$ were the support vectors shown in Fig. 1 . The kernel function $K\left(x, x_{i}^{S V}\right)$ can implicitly compute a nonlinear mapping $\quad x \rightarrow \Phi(x)$ and subsequent scalar multiplication $\Phi(x)^{T} \Phi\left(x_{i}^{S V}\right)$ in the mapped space in one step.

Using appropriate kernels, SVM offered an efficient tool for flexible classification with a highly nonlinear decision boundary. To date, the kernel generally used in BCI research was the Gaussian or radial basis function (RBF) kernel

$$
K\left(x, x_{i}\right)=\exp \left(\frac{\left\|x-x_{i}\right\|^{2}}{2 \sigma^{2}}\right)
$$

For SVM using the RBF kernel, two parameters had to be fixed in advance: the trade-off parameter $\mathrm{C}$ and the kernel parameter $\sigma$.They could be optimized for an optimal generalization performance in the traditional way, by using an independent test set or $\mathrm{n}$-fold cross-validation.

For multiclass SVM, couple of approaches is used usually for classification, one is to combine several binary classifiers and the other is to consider all the classes in one big optimization problem. In this study Binary Tree of SVM (BTS), which combines support vector machine and binary tree, with advantage on solving multiclass problems, was used here to identified four kinds of imaginary movements,

\section{DAta Processing Results}

The raw motor imagery EEG data is from the nonstationary BCI Competition 2005 database of Austrian Graz scientific and technical university. In the experiment, The subject sat in a relaxing chair with armrests, The task was to 
perform imagery left hand, right hand, foot or tongue movements according to a cue. As shown in Fig. 2 [14], all the experiment consists of three sections. after trial begin, the first $2 \mathrm{~s}$ were quite, at $\mathrm{t}=2 \mathrm{~s}$ an acoustic stimulus indicated the beginning of the trial, and a cross "+" is displayed; then from $\mathrm{t}=3 \mathrm{~s}$ an arrow to the left, right, up or down was displayed for 1 $\mathrm{s}$; at the same time the subject was asked to imagine a left hand, right hand, tongue or foot movement, respectively, until the cross disappeared at $\mathrm{t}=7 \mathrm{~s}$. Three subjects included $(\mathrm{k} 3 \mathrm{~b}$, $\mathrm{k} 6 \mathrm{~b}, 11 \mathrm{~b}$ ).

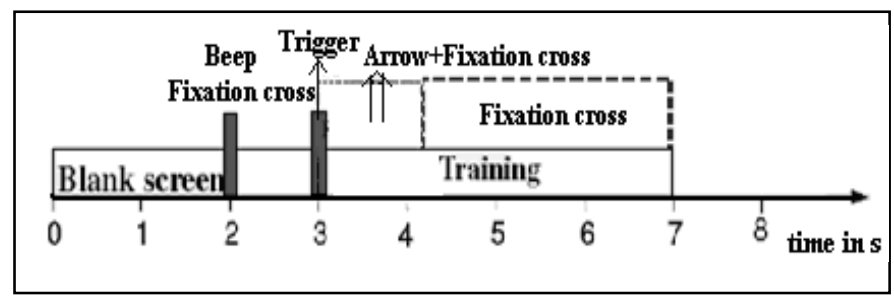

Figure 2. The sequence of the experiment

\section{A. ERD / ERS signals analysis}

Fig. 3 shows the PSD curve for the four types of imaginary movement patterns at $\mathrm{C} 3, \mathrm{Cz}, \mathrm{C} 4$ before ICA. EEG hands with $8 \sim 15 \mathrm{~Hz}, 20 \sim 28 \mathrm{~Hz}$ from all the 3 channels have been analyzed in this project while just 11 and $12 \mathrm{~Hz}$ were illuminated for the limited space.

The figures show that:

- The most significant ERD/ERS for left and right hand motor imageries appears at the contra-lateral channels. While C4 for left hand and C3 for right hand.

- Represent as ERD, the most modulated channel for foot imagery, is $\mathrm{Cz}$, and is weaker than that of hands.

- With the same modulated channel, , Tongue imagery expresses strong ERS on $\mathrm{Cz}$.

The main difficulty to identify the 4 types of imaginary movement is the distinction between foot and tongue movements.

Fig. 4 shows the PSD curves for 4 types of imaginary movement potentials after ICA spatial filter

Compare the 4 kinds of PSD curves:

- Left hand movement: the components of 24, 33, 35 with frequency of $11 \mathrm{~Hz}, 20 \mathrm{~Hz}, 25 \mathrm{~Hz}$ and $26 \mathrm{~Hz}$ represent ERD.

- $\quad$ Right hand movement: the components of 18, 27, 29 with frequency of $11 \mathrm{~Hz}, 12 \mathrm{~Hz}, 27 \mathrm{~Hz}$ and $29 \mathrm{~Hz}$ are ERD.

- $\quad$ Foot movement: the component of 21,30,32,41 with 8 $\mathrm{Hz}, 29 \mathrm{~Hz}$ are ERD, the components of 18, 19, 29, 35 with $26 \mathrm{~Hz}, 27 \mathrm{~Hz}$ are ERS.

- $\quad$ Tongue movement: the component of 29, 39, 40, 47 with $11 \mathrm{~Hz}, 29 \mathrm{~Hz}$ are ERD, the component of 18, 35, 48,55 with $12 \mathrm{~Hz}, 20 \mathrm{~Hz}, 25 \mathrm{~Hz}, 27 \mathrm{~Hz}, 29 \mathrm{~Hz}$ are ERS.

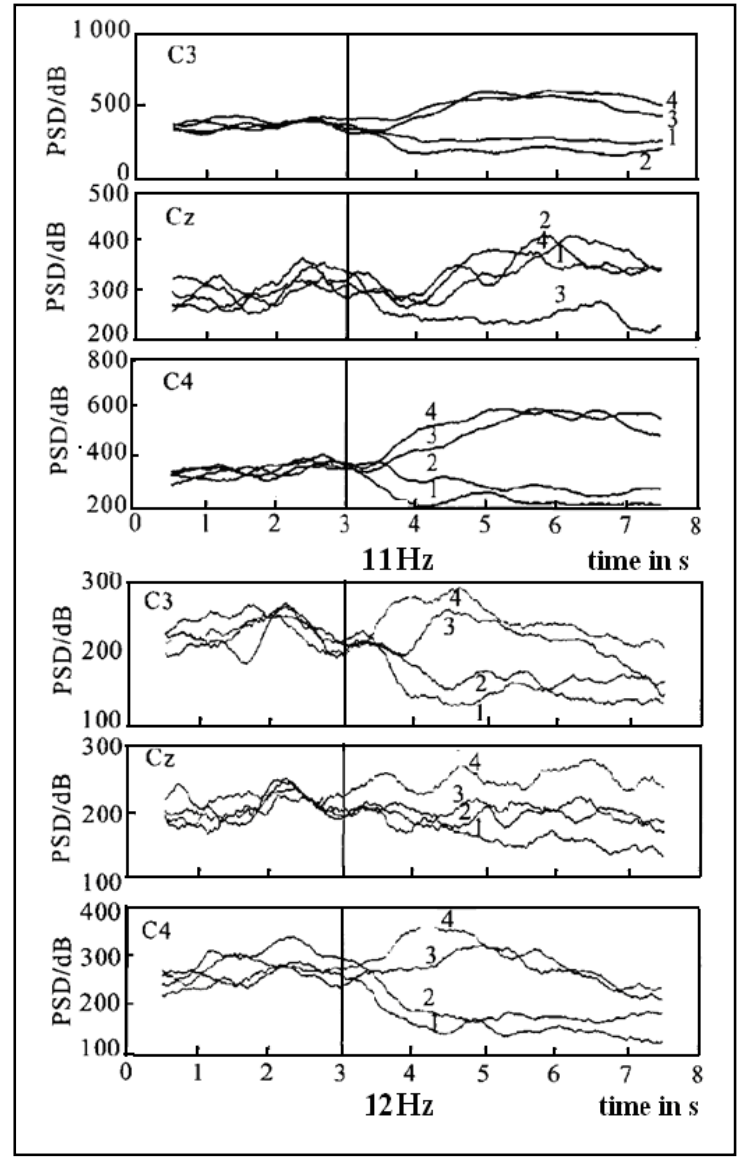

Figure 3. PSD curves of 4 types of imaginary movement potentials at electrode positions $\mathrm{C} 3, \mathrm{Cz}$ and $\mathrm{C} 4$. 1,2,3,4 respectively left hand, right hand, feet, tongue imaginary movement, $3 \mathrm{~s}$ is the beginning of the movements

\section{B. FeatureExtraction and classification for imaginary movement}

According to the results of ERD/ERS response analyzed above, following frequency bands were selected for various channels and the components:

- $\quad$ Left hand movement: $\mathrm{C} 4$ at $11 \mathrm{~Hz}, 20 \mathrm{~Hz}, 21 \mathrm{~Hz}, 22 \mathrm{~Hz}$, $23 \mathrm{~Hz}, 26 \mathrm{~Hz}, 27 \mathrm{~Hz}, 28 \mathrm{~Hz} ; 24,33,35,44$ component at $11 \mathrm{~Hz}, 20 \mathrm{~Hz}, 25 \mathrm{~Hz}, 26 \mathrm{~Hz}$ (they are all ERD).

- $\quad$ Right hand movement: $\mathrm{C} 3$ at $11 \mathrm{~Hz}, 12 \mathrm{~Hz}, 22 \mathrm{~Hz}, 23 \mathrm{~Hz}$, $26 \mathrm{~Hz}, 27 \mathrm{~Hz}, 28 \mathrm{~Hz}, 29 \mathrm{~Hz} ; 18,27,29,38$ component at $11 \mathrm{~Hz}, 12 \mathrm{~Hz}, 27 \mathrm{~Hz}, 29 \mathrm{~Hz}$ (they are all ERD too).

- $\quad$ Foot movement: $\mathrm{Cz}$ at $9 \mathrm{~Hz}, 11 \mathrm{~Hz}, 28 \mathrm{~Hz}, 29 \mathrm{~Hz}$; $\mathrm{C} 4$ at $8 \mathrm{~Hz}, 26 \mathrm{~Hz}, 27 \mathrm{~Hz} ; \mathrm{C} 3$ at $21 \mathrm{~Hz}, 22 \mathrm{~Hz}, 26 \mathrm{~Hz}, 27 \mathrm{~Hz}$; $21,30,32,41$ component at $8 \mathrm{~Hz}, 29 \mathrm{~Hz} ; 18,19,29,35$ component at $15 \mathrm{~Hz}, 25 \mathrm{~Hz}, 26 \mathrm{~Hz}, 27 \mathrm{~Hz}$.

- Tongue movement: $\mathrm{C} 3$ at $12 \mathrm{~Hz} ; \mathrm{Cz}$ at $12 \mathrm{~Hz}, 20 \mathrm{~Hz}$, $23 \mathrm{~Hz}, 24 \mathrm{~Hz}, 25 \mathrm{~Hz}, 27 \mathrm{~Hz}, 28 \mathrm{~Hz}, 29 \mathrm{~Hz} ; \mathrm{C} 4$ at $12 \mathrm{~Hz}$, $15 \mathrm{~Hz}, 23 \mathrm{~Hz} ; 29,39,40,47 \mathrm{component}$ at $11 \mathrm{~Hz} ; 18,35$, 48,55 component at $12 \mathrm{~Hz}, 20 \mathrm{~Hz}, 27 \mathrm{~Hz}, 29 \mathrm{~Hz}$. 


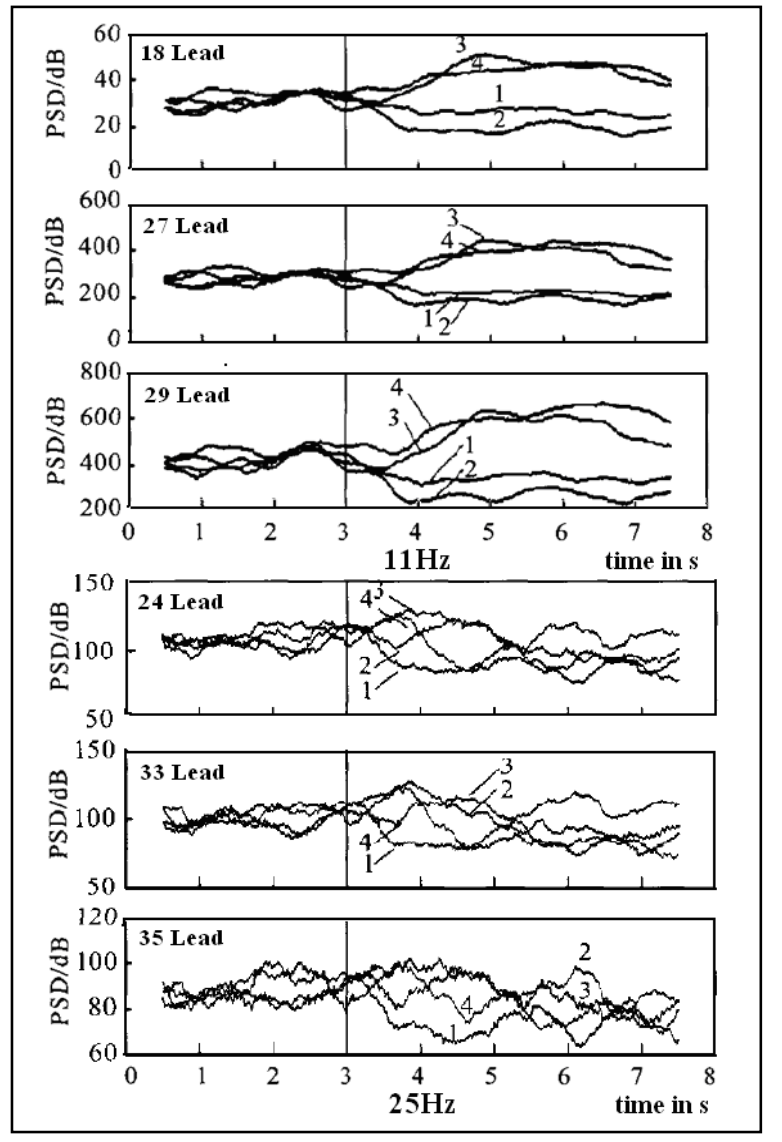

Figure 4. PSD curves of 4 types of imaginary movement potentials at certain characteristic electrode after ICA spatial filter. 1,2,3,4 respectively left hand, right hand, feet, tongue movement, $3 \mathrm{~s}$ is the beginning of the movements

Here, the ERD / ERS coefficient derived from power spectrum was defined as below:

$$
\mathrm{ERD} / \mathrm{ERS}=\frac{\mathrm{REP}_{\mathrm{t}_{1}}-\mathrm{REF}_{\mathrm{t}_{2}}}{\mathrm{REF}_{\mathrm{t}_{2}}} \times 100 \%
$$

where REP is the power spectral density in t1 after the imaginary movements, REF is the power spectral density in $\mathrm{t} 2$ before the imaginary movements, $\mathrm{t} 1$ and $\mathrm{t} 2$ must be consistent for all the trails, and were confirmed in this research as $t 1=3.1 \mathrm{~s}$ $\sim 4.6 \mathrm{~s}$ (stimulation occurred at the 3 th second), $\mathrm{t} 2=0.5 \mathrm{~s} \sim 2 \mathrm{~s}$.

\section{1) ERD/ERS coefficient Method}

The ERD / ERS coefficients for the selected componentfrequency bands with different imaginary patterns here were calculated respectively firstly. And then, average all the absolute coefficients. The movement corresponding to the largest of the four values is considered as the target that imagination movement occurred.

The prediction results are shown in Table 1. It displays that the accuracy of left and right hand is much higher than that of foot and tongue. Among of all the three data sets, the group $\mathrm{k} 3 \mathrm{~b}$, with less signal interference, performed better than the others.
TABLE I. THE EDICTION CURACY RATE BY ERD / ERS COEFFICIENT METHOD

\begin{tabular}{|l|l|l|l|}
\hline Movement & \multicolumn{1}{|c|}{ k3b } & \multicolumn{1}{|c|}{ k6b } & l1b \\
\hline Left hand (\%) & 79.4 & 40.2 & 38.9 \\
\hline Right hand (\%) & 78.2 & 40.8 & 37.5 \\
\hline Foot (\%) & 55.6 & 26.4 & 22.2 \\
\hline Tongue (\%) & 48.5 & 13.3 & 16.7 \\
\hline Total (\%) & 65.4 & 30.4 & 28.8 \\
\hline
\end{tabular}

\section{2) ERD / ERS coefficient + SVM Classification}

With ERD/ERS coefficients, 45 trails were used to train the SVM and the others are for testing, results are in Table 2.

Comparing with the results in Table 1, the accuracy improved significantly after classifying by SVM.

TABLE II. THE PREDICTION ACCURACY RATE BY ERD / ERS COEFFICIENT + SVM CLASSIFICATION

\begin{tabular}{|l|l|l|l|}
\hline Movement & \multicolumn{1}{|c|}{ k3b } & \multicolumn{1}{c|}{ k6b } & l1b \\
\hline Left hand (\%) & 89.6 & 65.2 & 64.4 \\
\hline Right hand (\%) & 85.4 & 60.3 & 58.7 \\
\hline Foot (\%) & 69.5 & 39.2 & 36.0 \\
\hline Tongue (\%) & 58.6 & 33.6 & 32.3 \\
\hline Total (\%) & 75.8 & 48.3 & 47.9 \\
\hline
\end{tabular}

\section{3) ICA filter + SVM Classification}

To consist of most prominent ERD/ERS bands and the least signals interference for modulated components, the frequency bands $9 \sim 12 \mathrm{~Hz}, 20 \sim 29 \mathrm{~Hz}$ are chosen here for ICA-SVM classification here. The results shown in Table 3 indicate that the accuracy between $77.6 \%$ and $91.4 \%$ totally reached a higher level than those of ERD/ERS coefficients and ERD/ERS-SVM methods.

TABLE III. THE PEDICTION ACCURACY RATE BY ICA FILTER + SVM CLASSIFICATION

\begin{tabular}{|l|l|l|l|}
\hline \multicolumn{1}{|c|}{ Group } & \multicolumn{1}{|c|}{ k3b } & \multicolumn{1}{|c|}{ k6b } & l1b \\
\hline Movement & & & \\
\hline Reft hand (\%) & 97.1 & 88.2 & 86.4 \\
\hline Foot (\%) & 95.3 & 85.6 & 80.6 \\
\hline Tongue (\%) & 88.5 & 78.9 & 74.2 \\
\hline Total (\%) & 84.9 & 72.1 & 69.3 \\
\hline
\end{tabular}

\section{CONCLUSION}

The results show that ICA-SVM is an effectiveness method to classify different imagining movement patterns. It is remarkable that combination of ICA-based signal extraction algorithm and SVM-based classification method that based on ERD/ERS response for four different imaginary patterns achieved a high accuracy rate. This implies that such EEG signal processing method may be utilized in a BCI operated simply by motor imagery. 


\section{ACKNOWLEDGMENT}

This research was supported by National Natural Science Foundation of China (No.30970875, No.90920015 and No. 60501005), Joint Project of National Natural Science Foundation of China-Royal Society of Edinburgh of UK (No. 30910494/C1009), The National High Technology Research and Development Program of China (No.2007AA04Z236), Intentional Cooperation Key Program (No.08ZCGHHZ00300) and Biomedical Engineering Key Program (No. 07ZCKFSF01300) of Tianjin Science Technology Support Plan.

\section{REFERENCES}

[1] Wolpaw JR, Birbaumer N, McFarland DJ, Pfurtscheller G, and Vaughan T M. "Brain-computer interfaces for communication and control," Clin. Neurophysiol., vol. 113, pp. 767-791, 2002.

[2] Leeb R, Lee F, Keinrath C, Scherer R, Bischof H, Pfurtscheller G, "Brain-computer communication: motivation, aim, and impact of exploring a virtual apartment," IEEE Trans. Neural Syst. Rehabil. Eng., vol. 15, no. 4, pp. 473-482, December 2007.

[3] Nijboer F, Furdea A, Gunst I, Mellinger J, McFarland DJ, Birbaumer N, et al., "An auditory brain-computer interface (BCI)," J. Neurosci. Methods, vol. 167, no. 1, pp. 43-45, January 2008.

[4] Schalk G, Brunner P, Gerhardt LA, Bischof H, Wolpaw JR, "Braincomputer interfaces (BCIs): detection instead of classification" J. Neurosci. Methods, vol. 167, no. 1, pp. 51-62, January 2008.

[5] Hsu WY, "EEG-based motor imagery classification using neuro-fuzzy prediction and wavelet fractal features," J. Neurosci. Methods, April 2010 .

[6] Pei XM, Zheng CX, Xu J, Bin GY, Wang HW, "Multi-channel linear descriptors for event-related EEG collected in brain computer interface," J. Neural Eng., vol. 3, no. 1, pp. 52-58, February 2006.

[7] Neuper C, Scherer R, Wriessnegger S, Pfurtscheller G, "Motor imagery and action observation: modulation of sensorimotor brain rhythms during mental control of a brain-computer interface," Clin. Neurophysiol., vol. 120, no. 2, pp. 239-247, February 2009.

[8] Pfurtscheller G, Neuper C, Flotzinger D, Pregenzer M, "EEG-based discrimination between imagination of right and left hand movement," Electroencephalogr. Clin. Neurophysiol., vol. 103, no. 6, pp. 642-651, December 1997.

[9] Lemm S, Schäfer C, Curio G, "BCI Competition 2003--Data set III: probabilistic modeling of sensorimotor mu rhythms for classification of imaginary hand movements," IEEE Trans. Biomed Eng., vol. 51, no. 6, pp. 1077-1080, June 2004.

[10] Li K, Sankar R, Arbel Y, Donchin E, "Single trial independent component analysis for P300 BCI system," Conf. Proc. IEEE Eng. Med. Biol. Soc., pp. 4035-4038, 2009.

[11] Patel A, Alotaibi F, Blume WT, Mirsattari SM, "Independent component analysis of subdurally recorded occipital seizures," Clin. Neurophysiol., vol. 119, no. 11, pp. 2437-2446, November 2008.

[12] Wan BK, Zhou ZX, Ming D, Qi HZ, "Detection of ERD/ERS Signals Based on ICA and PSD," J. Tianjin Univ, vol. 41, no. 11, pp. 1383-1390, November 2008.

[13] Demirer RM, Ozerdem MS, Bayrak C, "Classification of imaginary movements in ECoG with a hybrid approach based on multi-dimensional Hilbert-SVM solution," J. Neurosci. Methods, vol. 178, no. 1, pp. 214218, March 2009.

[14] Xu Q, Zhou H, Wang Y, Huang J, "Fuzzy support vector machine for classification of EEG signals using wavelet-based features," Med. Eng. Phys., vol. 31, no. 7, pp. 858-865, May 2009. 